Cite this: J. Mater. Chem. B, 2014, 2, 2282

Received 17th September 2013 Accepted 5th February 2014

DOI: $10.1039 / \mathrm{c} 3 \mathrm{tb} 21280 \mathrm{~g}$

www.rsc.org/MaterialsB

\section{Development and characterisation of a new bioink for additive tissue manufacturing $\dagger$}

\author{
Ferry P. W. Melchels, ${ }^{\text {ab }}$ Wouter J. A. Dhert, ${ }^{\text {ac }}$ Dietmar W. Hutmacher ${ }^{\text {bde }}$ \\ and Jos Malda abc
}

\begin{abstract}
Additive manufacturing forms a potential route towards economically viable production of cellular constructs for tissue engineering. Hydrogels are a suitable class of materials for cell delivery and 3D culture, but are generally unsuitable as construction materials. Gelatine-methacrylamide is an example of such a hydrogel system widely used in the field of tissue engineering, e.g. for cartilage and cardiovascular applications. Here we show that by the addition of gellan gum to gelatinemethacrylamide and tailoring salt concentrations, rheological properties such as pseudo-plasticity and yield stress can be optimised towards gel dispensing for additive manufacturing processes. In the hydrogel formulation, salt is partly substituted by mannose to obtain isotonicity and prevent a reduction in cell viability. With this, the potential of this new bioink for additive tissue manufacturing purposes is demonstrated.
\end{abstract}

\section{Introduction}

Additive manufacturing (AM) is an emerging class of technologies in which materials are joined layer upon layer to fabricate objects from three-dimensional (3D) model data. ${ }^{1}$ Advantages of $\mathrm{AM}$ as opposed to traditional subtractive manufacturing technologies are reduced product development times (rapid prototyping, rapid manufacturing), freedom of design (customisation) and limited material waste. ${ }^{2}$ These features make AM attractive for medical applications, as instruments, implants, devices and time in the clinic are costly and scarce. Moreover, AM technology platforms can be integrated in personalised medicine concepts, which is a rapidly increasing area of interest in modern medicine. AM techniques are being applied clinically to personalise treatment concepts, such as for fabricating anatomical models for pre-operative planning ${ }^{3}$ or for guides used intra-operatively for resections, drilling ${ }^{4}$ and reconstructing. ${ }^{5} \mathrm{AM}$ and specific bioprinting do form a potential route towards engineering of living (containing viable cells)

${ }^{a}$ Department of Orthopaedics, University Medical Center Utrecht, PO Box 85500, 3508 GA Utrecht, The Netherlands. E-mail: j.malda@umcutrecht.nl; Fax: +31 30251 0638; Tel: +31887558078

${ }^{b}$ Institute for Health and Biomedical Innovation, Queensland University of Technology, 60 Musk Avenue, Kelvin Grove, QLD 4059, Australia.E-mail: dietmar.hutmacher@qut. edu.au; Fax: +61 73138 6030; Tel: +61 731386077

'Department of Equine Sciences, Faculty of Veterinary Sciences, Utrecht University, Yalelaan 112, 3584 CM Utrecht, The Netherlands

${ }^{d}$ George W. Woodruff School of Mechanical Engineering, Georgia Institute of Technology, Atlanta, GA, USA

${ }^{e}$ Institute for Advanced Studies, Technical University Munich, Munich, Germany

$\dagger$ Electronic supplementary information (ESI) available. See DOI: $10.1039 / \mathrm{c} 3 \mathrm{tb} 21280 \mathrm{~g}$ or tissue inductive constructs for regenerative medicine as the current paradigm of manually seeding cells onto prefabricated scaffolds often does not allow for recapitulation of the complex architecture of native tissues; neither can it be scaled up to meet clinical demands in an economically and logistically feasible manner. ${ }^{6}$

Bioprinting uses a computer controlled printing device to accurately deposit cells and/or biomaterials in the form of a hydrogel into precise forms, shapes and volumes with the goal to build anatomically organised structures. Several technology platforms are available such as extrusion printing, microvalve printing, inkjet printing and laser-assisted printing. ${ }^{7}$ Whereas the latter two are unsurpassable in terms of spatial resolution, the former two are generally more suited for building up larger 3D structures and may therefore have more clinical relevance for tissue engineering. However, an important limitation in clinical translation of these techniques is that it requires specialised (bio)material platforms, which bear the bioprinting required for physiochemical as well as biological properties. When focussing on extrusion printing of 3D structures, there are a number of common hydrogel formulations that hold potential for use as the so called bioink. These are based on biopolymers of natural origin such as collagen, ${ }^{8}$ gelatine, hyaluronic acid, ${ }^{9}$ chitosan $^{10}$ and alginate, ${ }^{11}$ or synthetic origin such as poly(ethylene glycol) $)^{12}$ and/or poly( $N$-hydroxypropylmethacrylacrylamide lactate). ${ }^{13}$ Bioinks in the form of hydrogels require contrasting properties of a hydrogel material. For manufacture, highly viscous gel precursors, that can be swiftly cross-linked into stiff and strong gels, are favoured for constructing high-fidelity structures. ${ }^{14}$ However, cells are preferably encapsulated in soft gels with maximal water content and low 
crosslink densities, in which their metabolism, proliferation and migration are only minimally restricted. ${ }^{15}$ Unfortunately, bioinks which fulfil this catalogue of requirements are currently not readily available. Plotted cell-free hydrogel structures with very well defined (pore) architecture have been reported, ${ }^{16,17}$ however, inclusion of cells in the fabrication process generally comes at the cost of shape fidelity. ${ }^{11,18}$ Hence, we postulate that the current lack of suitable hydrogel systems for additive tissue manufacturing and specific bioprinting forms the major bottleneck for the further maturation of this field. ${ }^{6}$ Therefore, in the current paper we describe the development of a novel bioink, including the characterisation of the properties that govern its behaviour in bioprinting processes. We report on the design and characterisation of a gelatine-based biomaterial platform that is suitable for the fabrication of well-defined hydrogel structures (precise forms, shapes and volumes) using an extrusion-based bioprinting process that enables the building of highly reproducible 3D custom-shaped tissue constructs.

Gelatine, a protein-based hydrogel, is a well-established biomaterial and is favoured for its wide availability, inherent cell adhesion domains, low immunogenicity and enzymatic degradability. ${ }^{19}$ Gelatine can be functionalised with methacrylamide groups, ${ }^{20}$ enabling irreversible cross-linking (e.g., by UV-irradiation in the presence of a photo-initiator) for shape preservation under physiological conditions. For these reasons, gelatine-methacrylamide (gelMA) is receiving increasing interest in the biofabrication and tissue engineering fields. ${ }^{21-25}$ Its low viscosity and slow thermal gelation make gelMA suitable for casting and micro-moulding, ${ }^{21}$ however restrict its application for bioprinting. Extrusion printing of gelMA has been successfully performed with ultra-precise control of ink and nozzle temperature, combined with cooling of the build platform (to $\sim 5{ }^{\circ} \mathrm{C}$ ).$^{26}$ Here we demonstrate how the ability to manufacture gelatine-based structures and constructs can be greatly facilitated and improved by other means than critical hardware modifications or by increasing the polymer concentration or crosslink density. We modified the rheological behaviour of gelatine gels by the addition of the high-molecular weight polysaccharide gellan gum at tailored salt concentrations, thereby inducing pseudo-plastic behaviour and yield stress in addition to the thermosensitivity inherent to gelatine.

\section{Results and discussion}

Gellan gum is a hydrolysable bacterial polysaccharide, which has FDA approval as a food additive and is also being investigated for its application in tissue engineering. ${ }^{27}$ The low-acyl variant is the most commonly encountered gellan gum, which on its own forms brittle gels in the presence of cations (of any valence) under physiological conditions. As the gellan chains bind through ionic cross-linking, the ionic interaction and gel strength are strongly dependent on the salt concentration. Here, we use small concentrations of gellan gum with tuned salt concentrations added to gelMA solutions to induce desirable rheological behaviour for gel plotting. These desired rheological phenomena include pseudo-plasticity (a form of shear thinning) and yield stress (Fig. 1A). The cations in the hydrogel precursor
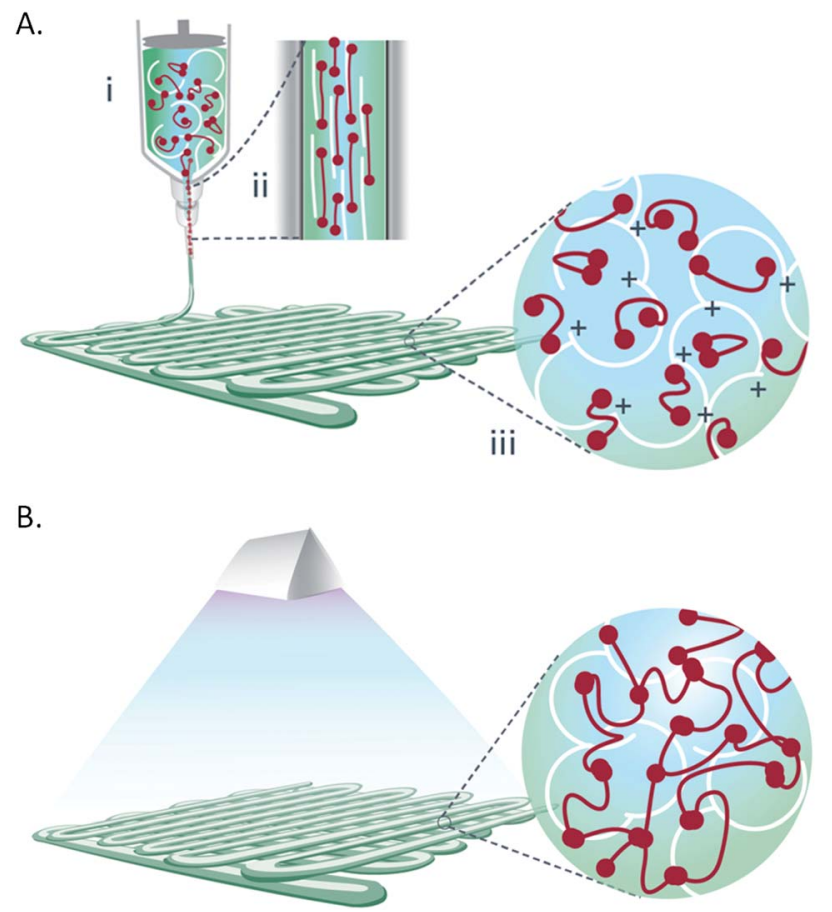

Fig. 1 Schematic representation of gelMA/gellan plotting (A) followed by UV curing (B). In the syringe the gellan chains (in white) form a temporary network in the presence of cations, and induce gel-like behaviour (i). Upon dispensing through a nozzle, the temporary network is broken up by shear forces and all polymer chains align resulting in a reduction of the viscosity (ii). Directly after removal of shear stress, the temporary network is restored and the plotted filament solidifies instantly (iii), after which the slower thermal gelation of gelMA further increases the stability of the printed bars and struts. Upon UV exposure the gelMA chains (in red) form a permanent polymer network (B). Panel $1 \mathrm{~A}$ reprinted with permission from ref. 37. Copyright (2013) John Wiley and Sons.

solution - both dissolved sodium ions and protonated groups on the gelMA chains - form ionic crosslinks between the gellan chains, inducing gel-like behaviour below $42^{\circ} \mathrm{C}(\mathrm{i}$, concentrationdependent). Upon application of shear by dispensing, the physical network is broken up and the flow is initiated. As the solution passes through the nozzle, the increased shear rates provoke alignment of the polymer chains which results in reduction of its viscosity (pseudo-plasticity), facilitating the dispensing (ii). As the shear loading is removed after exiting the nozzle, the ionic network is reformed instantly (iii) resulting in deposition of a solid hydrogel filament. This preserves the shape of the fabricated construct, which is further reinforced as the gelMA component forms additional physical crosslinks by forming triple helices below $32{ }^{\circ} \mathrm{C}$ (at $10 \%$ concentration). Finally, the gelMA is chemically crosslinked by UV-initiated crosslinking (Fig. 1B), thereby resulting in irreversible shape fixation.

These rheological phenomena induced by the transient physical crosslinking of gellan gum were further investigated (Fig. 2). The viscosity of $10 / 0.75 \%$ gelMA/gellan mixtures in water was 1 order of magnitude higher than that of the individual components, which can be explained from the interaction between the deprotonated (negatively charged) glucuronic acid residues on 
A
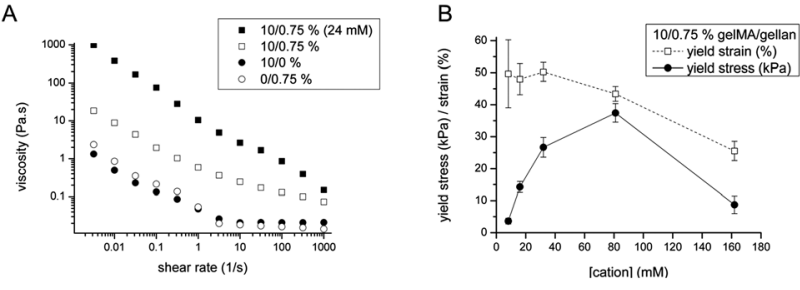

C
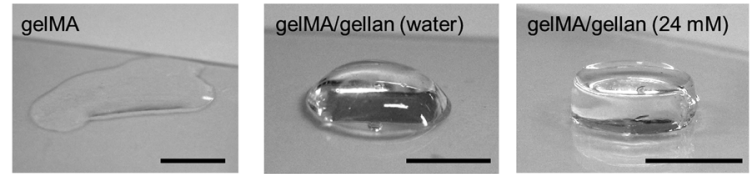

Fig. 2 Rheological behaviour of gelMA/gellan at $37{ }^{\circ} \mathrm{C}$. (A) The viscosity of $10 / 0.75 \%$ gelMA/gellan in water is $1-3$ orders of magnitude higher than that of the individual components, depending on the salt concentration. (B) Cation concentration dependency of yield stress and yield strain for 10/0.75\% gelMA/gellan. (C) Uncured 10\% gelMA discs at $37{ }^{\circ} \mathrm{C}$ spread in the absence of gellan, flow to blobs with $0.75 \%$ gellan in water but keep their shape at $16 \mathrm{mM}$ cations due to yield stress. Scale bars are $5 \mathrm{~mm}$.

the gellan gum chains and the protonated (positively charged) lysine groups on the gelMA chains (Fig. 2A). The addition of small quantities of monovalent cations further increased the viscosity by almost 2 orders of magnitude for low shear rates representative of sagging $\left(10^{-2} \mathrm{~s}^{-1}\right)$, but less than 1 order of magnitude for shear rates representative of plotting $\left(10^{2} \mathrm{~s}^{-1}\right)$. The pseudo-plasticity is apparent, with a flow index of 0.33 when fitting the data to the Herschel-Bulkley model ${ }^{28}$ in which $n=1$ designates a Newtonian fluid, and $n<1$ indicates pseudo-plasticity or shear-thinning. Next, the yield stress was assessed. Whereas solutions of gelMA in water ( $10 \%$ ) spread completely, the addition of $0.75 \%$ gellan gum reduced the flow considerably to formation of a slightly spread droplet (Fig. 2C). However, in the presence of $24 \mathrm{mM}$ added cations, the casted disc shape was fully retained. The absence of flow is evidenced by sharp edges of the gel disc, which means a yield stress is present that is stronger than the sum of surface tension and gravity, ensuring shape preservation.

The yield stress was quantified by performing compression tests on an uncured cast cylindrical gel specimen at $37^{\circ} \mathrm{C}$, and appeared strongly dependent on the salt concentration (Fig. 2B). In this study, we used a range of dilutions of phosphate buffered saline (PBS), which has a concentration of monovalent cations $\left(\mathrm{Na}^{+}\right.$plus $\left.\mathrm{K}^{+}\right)$of $162 \mathrm{mM}$. Where the gel mixture in water did not exhibit a compressive yield stress within the detection limit of our setup, the same composition at $8.1 \mathrm{mM}$ salt concentration (equivalent to $20 \times$ diluted PBS) showed a clear yield point at $3.4 \pm 0.8 \mathrm{kPa}$. The yield stress shows a steep increase with cation concentration up to $34.3 \pm$ 2.7 for $81 \mathrm{mM}$, with the yield strain gradually decreasing from approximately 50 to $43 \%$. At $162 \mathrm{mM}$ (undiluted PBS) however, both yield stress and strain are considerably decreased as the mixture phase-separates due to too strong interactions between the gellan gum chains.

The obtained rheological properties of $10 / 0.75 \%$ gelMA/gellan at $24 \mathrm{mM}$ salt enable plotting of gel structures using an adapted fused deposition modelling (FDM) method. Continuous filaments are deposited by moving the collection platform under the nozzle at close proximity. Both stage and piston movement are computer controlled from the same source code. As a result, strands with diameters close to the diameter of the used nozzle can be deposited. To achieve this, the translational speed of the collecting stage has to match the flow velocity of the gel precursor through the nozzle. The flow velocity $v$ $\left(\mathrm{mm} \mathrm{min}^{-1}\right)$ can be derived from the volumetric flow rate $\phi$ $\left(\mathrm{mm}^{3} \mathrm{~min}^{-1}\right)$ and the diameter of the nozzle $D(\mathrm{~mm})$ :

$$
v=\frac{4 \phi}{\pi D^{2}}
$$

Thicker filaments can be easily obtained by increasing the dispensing rate relative to the stage speed, allowing the extruded filament to 'swell'. In this way a wide range of strand diameters can be obtained with a single nozzle gauge (Fig. 3A and B). This enables to select between plotting at high resolution (with thin filaments) or at high throughput (with thick filaments), even within different regions of a single construct. The lower limit for the filament diameter corresponds approximately to the inner diameter of the nozzle $(0.21 \mathrm{~mm}$ for $27 \mathrm{G})$; as the gel filament is composed mainly of water and there is only a low extent of entanglement between the polymer chains, it tends to break when the stage speed exceeds the flow velocity considerably.

3D structures can be plotted by depositing gel filaments in a layer-by-layer fashion. Hereto, the distance between the nozzle and the collection platform is increased by a set value (typically $60-80 \%$ of the filament diameter to ensure proper fusion) after
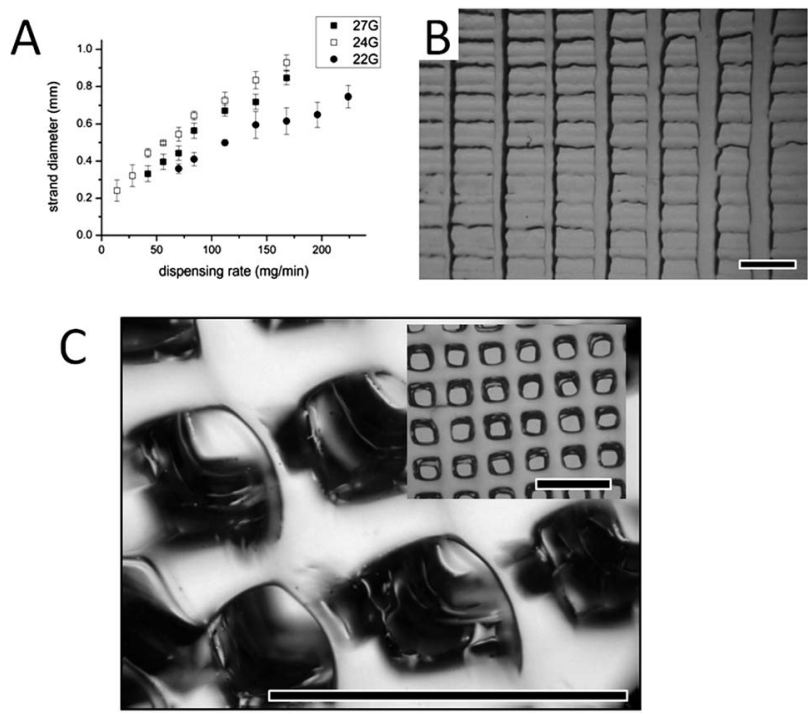

Fig. 3 Plot of grid structures from 10/0.75\% gelMA/gellan at $24 \mathrm{mM}$ salt and $500 \mathrm{~mm} \mathrm{~min}^{-1}$ stage speed. (A) The strand diameter strongly depends on the dispensing rate, and to a lower extent on the nozzle gauge used. (B) Photograph of the grid plotted with 27G nozzle with increasing dispensing rate (left to right). (C) 10 layer high grid structure with $1 \mathrm{~mm}$ strand spacing (line centre to line centre) (D). Scale bars are $2 \mathrm{~mm}$. 
each layer is completed. Porous grid structures of 10 layer height were prepared in this manner, in which the individual filaments in different layers of the stack can be clearly distinguished (Fig. 3C). Choosing an appropriate step height is crucial in ensuring proper fusing of the deposited strand to the preceding layer to obtain a mechanically integer construct. When the $z$-step height is too large, drift occurs and the nozzle moves further away from the plotted structure with every subsequent layer, leading to filament interruptions and eventually, low fidelity constructs. However, when the $z$-step height is too small, the deposited layer can sink into the previous layer considerably which leads to loss of porosity and shape fidelity.

The dispensing rate, stage speed, inter-filament distance and layer step height can be matched to result in solid structures, as demonstrated by the pyramid and hemisphere in Fig. 4A and B. A video of the gel printing process is available online as ESI. $\uparrow$ Besides fabricating geometrical shapes such as these, in additive manufacturing it is possible to use data from medical scans to create patient-specific structures with anatomical shapes. ${ }^{5}$ Porosity can be introduced into plotted structures by reduction of the dispensing rate. For example, $30 \%$ reduction in the dispensing rate corresponds to $30 \%$ porosity (defined as the volume fraction of the void space in the structure) (Fig. 4C). In the context of additive tissue manufacturing, the main function of porosity would be to allow rapid transport of oxygen, nutrients and metabolites to and from cells encapsulated in the gels. ${ }^{29}$ In a porous construct (regardless of its overall dimensions) built from gel filaments of $0.4 \mathrm{~mm}$ diameter, the diffusion length between a cell and a perfused channel will be not

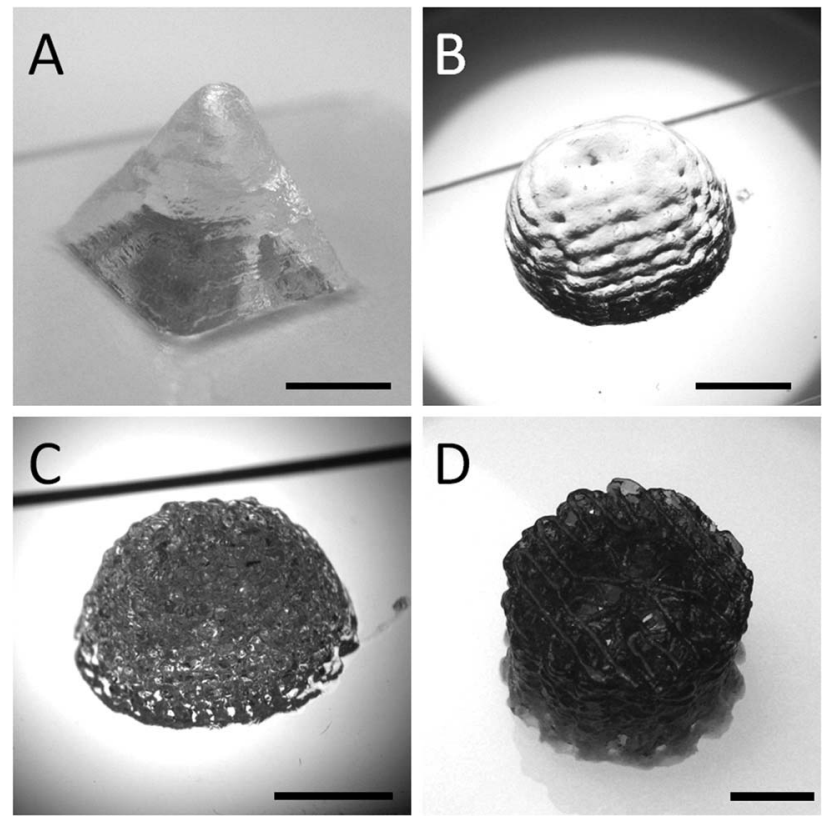

Fig. 4 Examples of plotted gelMA/gellan structures. (A and B) Solid pyramid and hemisphere plotted from 10/0.75\% gelMA/gellan at $24 \mathrm{mM}$ salt. (C) 30\% porous hemisphere plotted from 10/1.0\% gelMA/ gellan in $0.16 \mathrm{mM}$ salt. (D) Porous hollow cylinder plotted from 10/1.0\% gelMA/gellan in $0.16 \mathrm{mM}$ salt, after UV curing and immersion in blue dye. Scale bars are $5 \mathrm{~mm}$. more than $200 \mu \mathrm{m}$, similar to the maximum distance between cells and blood capillaries in native tissues. ${ }^{30}$ In this respect, gel constructs do not need to be as highly porous as scaffolds, in which a high porosity is required to provide space for seeded cells to proliferate and produce tissue. When using cell-laden gels the pore channels merely act as a perfusion network as the cells are inside the gel strands where they form tissue whilst remodelling the gel. The porous hollow cylinder prepared from 10/1.0\% gelMA/gellan at $16 \mathrm{mM}$ salt shown in Fig. 4D has been fabricated before in poly( $\varepsilon$-caprolactone) as a scaffold designed specifically for a tibial segmental defect, for which a pre-clinical model has been developed by our group. ${ }^{31}$ When fabricated using this hydrogel, structures could potentially contain boneforming cells, as well as blood-vessel forming cells to facilitate bone repair. Alternatively or additionally, growth factors could be released in a spatiotemporally controlled manner, by design of the architecture and the properties of the hydrogel (mesh size, degradation rate, inclusion of drug-releasing microspheres, etc.). Dimensions and structural parameters of gel structures prepared here were analysed using $\mu \mathrm{CT}$ (Fig. 5). Scans were performed directly after fabrication (on non-cured gels), as well as one day post-UV-curing, after overnight immersion in PBS. The diameter, height and weight of the freshly fabricated structures were slightly lower than those designed, caused by slight dehydration from evaporating water during fabrication (due to the lack of humidity control in the applied setup). A larger decrease was seen for the cured structures, which can be explained by shrinkage, a phenomenon commonly observed for UV-cured hydrogels. As the shrinkage is fairly isotropic (10\% in diameter, $11 \%$ in height and $23 \%$ in weight, which corresponds to $8 \%$ unidirectional shrinkage assuming the density to remain unchanged), it does not influence the shape of the fabricated construct and can be taken into account in the design. The porosity of the fabricated structures was close to the designed/ predicted porosity both before and after UV curing. Moreover, the $\sim 10 \%$ shrinkage was too low to induce a significant decrease in the average filament diameter and spacing, both of which were approximately $1 \mathrm{~mm}$ in all cases. The $\mu \mathrm{CT}$ visualisations in Fig. 5 demonstrate that the porous constructs were plotted with highly regular filaments almost comparable to fused deposition modelling of thermoplastics. Such high shape fidelity is rarely demonstrated with other hydrogel platforms.

\begin{tabular}{lllllll}
\hline & $\begin{array}{l}\text { diameter } \\
{[\mathrm{mm}]}\end{array}$ & $\begin{array}{l}\text { height } \\
{[\mathrm{mm}]}\end{array}$ & $\begin{array}{l}\text { weight } \\
\text { [g] }\end{array}$ & $\begin{array}{l}\text { porosity } \\
{[\%]}\end{array}$ & $\begin{array}{l}\text { filament } \\
\text { diameter } \\
{[\mathrm{mm}]}\end{array}$ & $\begin{array}{l}\text { filament } \\
\text { spacing } \\
{[\mathrm{mm}]}\end{array}$ \\
\hline designed & 18 & 11.6 & 1.39 & 52 & & \\
as fabricated & $16.4 \pm 0.5$ & $11.2 \pm 0.1$ & $1.33 \pm 0.02$ & $47 \pm 2$ & $0.98 \pm 0.2$ & $1.03 \pm 0.29$ \\
UV-cured & $14.7 \pm 0.4$ & $9.9 \pm 0.1$ & $1.03 \pm 0.02$ & $49 \pm 2$ & $1.08 \pm 0.24$ & $0.96 \pm 0.06$ \\
\hline
\end{tabular}
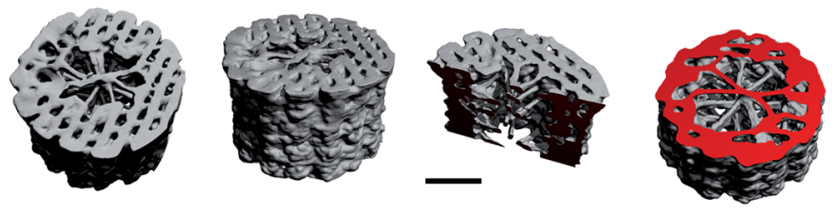

Fig. 5 Structural evaluation of plotted gel construct by $\mu \mathrm{CT}$. Quantitative parameters are of 3 structures directly after plotting, and after UV curing and PBS immersion. Scale bars represent $5 \mathrm{~mm}$. 
As application of this gel system for additive tissue manufacturing would imply cells to be incorporated in the hydrogel, it is important to create an isotonic environment as hypotonic conditions lead to osmotic stress, which could result in cell damage or possibly cell death through osmolysis. ${ }^{32}$ The salt concentrations used here to tailor the rheological properties of the gels are far below physiological levels (10-15\%), hence we investigated the use of the sugar mannose as a non-ionic temporary replacement for PBS to provide the cells with an isotonic environment during fabrication. Mannose has been used before as a regulator of tonicity, in the osmotic selection of mesenchymal stem cells from umbilical cord blood. ${ }^{33}$ The combined effect of replacing PBS by isotonic mannose and of adding $0.75 \%$ gellan to $10 \%$ gelMA on cell viability was investigated using the Alamar blue assay for metabolic activity (Fig. 6A). The hydrogel precursors mixed with cells were UV-cured and incubated overnight, after which the metabolic activity was assessed. The metabolic activity was normalised to that of 10\% gelMA in PBS. In this positive control, the absolute viability was determined using live/dead staining at $81 \pm 4 \%$, which is in the normal range for UV-cured gels. While the absence of PBS or mannose led to immediate cell death, no decrease in metabolic activity was observed for the gelMA/gellan in the mannose group. This indicates that neither the addition of gellan gum, nor the short-term lack of ions is detrimental for cell survival, as long as mannose is used to maintain isotonic conditions. This gives the user the freedom to independently tailor salt concentrations towards optimal rheological properties for biofabrication, without compromising cell viability. The metabolic activity was not reduced for up to 1 hour exposure to isotonic mannose-containing gel precursors in the absence of salts (data not shown). By comparison, fabrication of the $\sim 16 \times$ $11 \mathrm{~mm}$ construct shown in Fig. 5 takes 13 minutes. Constructs of $10 / 1.0 \%$ gelMA/gellan were successfully plotted with encapsulated cells (Fig. 7B). The addition of 0.5 million cells per $\mathrm{mL}$ did not visibly affect plotting characteristics. The plotted grid structure can be recognised in the fluorescent image showing live (green) and dead (red) cells in the image. Due to resuspension of cells in the gel at a supraphysiological temperature of $40-50{ }^{\circ} \mathrm{C}$ the viability is approximately $50 \%$, which is lower

A

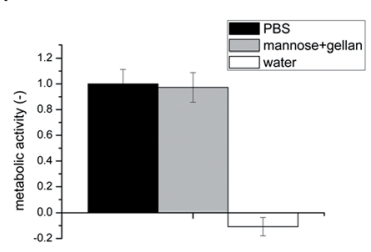

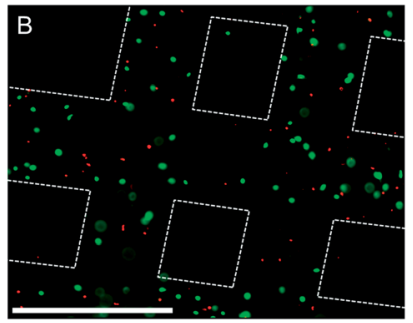

Fig. 6 Viability of cells encapsulated in gelMA/gellan gels. (A) Metabolic activity (24 h post-UV-curing) of cells encapsulated in 10/0.75\% gelMA/gellan in isotonic mannose $(0.30 \mathrm{M})$ or $10 \%$ gelMA in water, normalised to $10 \%$ gelMA in PBS. (B) Live/dead staining (4 d post-UVcuring) of the plotted cell-laden construct of $10 / 1.0 \%$ gelMA/gellan in isotonic solution of $16 \mathrm{mM} \mathrm{NaCl}$ and $0.27 \mathrm{M}$ mannose. Dashed white lines indicate pores in grid (scale bar is $1 \mathrm{~mm}$ ).

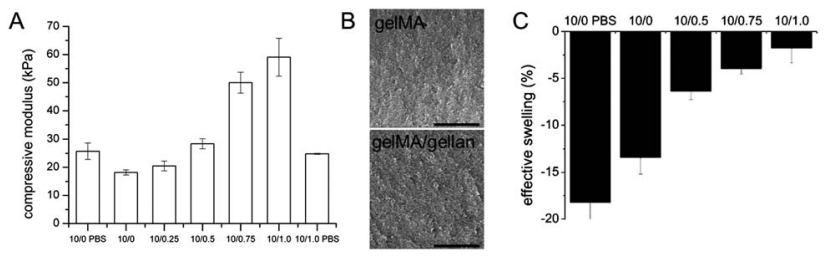

Fig. 7 Effect of gellan and mannose on the physical properties of UV-cured gelMA gels. (A) Compressive modulus for different gellan concentrations (in mannose) compared to gelMA or gellan alone (in PBS). (B) Scanning electron micrographs showing the microstructure of gelMA vs. gelMA/gellan gels (scale bars are $10 \mu \mathrm{m}$ ). (C) Effective swelling for different gellan concentrations (in mannose) compared to gelMA alone (in PBS)

than that in non-plotted controls that contained less gellan and no salt ( $81 \pm 4 \%$ viability). Current research efforts are aimed at decreasing the gelation temperature of gelMA/gellan mixtures, to enable resuspending cells at $37{ }^{\circ} \mathrm{C}$, thus decreasing cell death.

The physical properties of crosslinked gelMA-gels (10\%) with different concentrations of added gellan gum were evaluated. The gels were prepared in isotonic mannose, including $10 \%$ gelMA in PBS as a reference. The gel percentage (weight fraction of the gel precursor incorporated in the final network) was not affected by the gellan concentration or the medium; it was $78 \pm$ $4 \%$ across the whole range. The crosslinking reaction is incomplete (gel fraction $<100 \%$ ) as the crosslinking conditions UV exposure time in particular - have been optimised to obtain a mechanically robust gel while retaining high cell viability. ${ }^{25}$

The compressive modulus (tangent of stress-strain curve at $10-15 \%$ strain) was shown to increase progressively upon addition of gellan gum (Fig. 7A). Replacing PBS by mannose decreased the modulus from $26 \pm 3 \mathrm{kPa}$ to $18 \pm 1 \mathrm{kPa}$, which was counteracted by the addition of $0.5 \%$ gellan gum $(28 \pm 2$ $\mathrm{kPa}$ ). Adding more gellan increased the modulus considerably $(50 \pm 4 \mathrm{kPa}$ for $0.75 \%$ and $59 \pm 7 \mathrm{kPa}$ ). This can be explained by the formation of a physically crosslinked network of gellan that forms an interpenetrating network with the chemically crosslinked gelMA network. The physical network is formed as the UV cured gels are incubated in PBS and cations diffuse into the gel to crosslink the gellan. The compressive modulus of a $10 \%$ gelMA $+1 \%$ gellan gum gel (after UV-curing in mannose followed by PBS immersion) is slightly higher than the sum of those of a UV-cured 10\% gelMA gel and an ionic 1\% gellan gum gel: $59 \pm 7 \mathrm{kPa} v s .18 \pm 1 \mathrm{kPa}$ plus $24.8 \pm 0.2 \mathrm{kPa}$. This can be expected as the total crosslink density (with which the modulus scales linearly) is the sum of the crosslink densities of the two independent networks, plus the trapped entanglements which act as additional crosslinks.

Scanning electron microscopy revealed no change in the microstructure down to the micrometer level upon addition of $1 \%$ gellan gum to $10 \%$ gelMA (Fig. $7 \mathrm{~B}$ ). This indicates that these components are fully miscible at these concentrations, and remain mixed upon UV curing. Both images show uniform foams with pores of far below $1 \mu \mathrm{m}$ in size, implying isotropic homogeneous crosslinking. 
Shrinkage - common for UV-cured gelMA gels ${ }^{25}$ - was reduced upon replacing PBS by mannose, and further reduced as the gellan concentration increased (Fig. 7C). The shrinkage for 10/1.0 gelMA/gellan was only $2 \pm 2 \%$ (by weight) in these series, whereas the porous structures prepared from this composition shrunk by $23 \%$ (Fig. 5). The difference can be explained by a combination of UV attenuation into the much larger plotted structure, and inhibition of chain crosslinking polymerisation by oxygen. The gels used for characterisation of their physical properties were only $2 \mathrm{~mm}$ thick and UV cured in a fully enclosed mould, which minimises oxygen inhibition that free-radical polymerisations are sensitive to. ${ }^{34}$ The porous structures however were $11 \mathrm{~mm}$ tall and immersed in a solution of photo-initiator in PBS to avoid direct atmospheric exposure and depletion of initiator. However, the oxygen dissolved in the immersion medium would have had an inhibiting effect on the photo-initiated crosslinking, resulting in incomplete crosslinking. Assessing local methacrylamide conversion and stiffness throughout the structures are points for future investigation. Nevertheless, thermally stable, fully elastic hydrogel structures of the designed shapes were obtained with this fabrication and UV curing approach.

We presented a study of modifying a widely used hydrogel, namely gelatine-methacrylamide, for applications in additive manufacturing and specific bioprinting. In the young field of hydrogel-based bioprinting, the optimal range of material properties for gel plotting has not yet been established. However, for our system the best results were obtained in the same range of viscosity (of about 1 up to several Pas) as was previously reported for another biomaterial which was used as a bioink, namely sodium alginate. ${ }^{35}$ Even so 2 or $3 \%$ alginate solutions (with 0.9 or 2.0 Pas viscosity, respectively) can be printed, the spatial resolution and form stability of the strands and struts as well as the overall shape of a printed construct is far from being optimal. Having a sufficiently high viscosity is important for bioprinting, as it prevents surface tension-driven droplet formation at the nozzle tip, as well as collapse of the plotted structure. Conversely, higher viscosities imply higher shear stresses imposed on encapsulated cells, and mixing of cells into gel precursor solutions becomes more challenging without introducing air bubbles. The most practical way is to mix in the cells just above the gelation temperature of the gellan, when the viscosity is low (mPa range). The effect of short exposure to temperatures slightly above the body temperature (the gelation temperature of gellan at $1 \% \mathrm{w} / \mathrm{v}$ in PBS is $42{ }^{\circ} \mathrm{C}$ ) on cell viability and function is yet to be determined. However, the gelation temperature can be decreased by oxidation, ${ }^{36}$ which will be a point of further study for this application. Besides viscosity, the kinetics and timing of gelation are crucial for shape preservation. To our knowledge, the gelMA/gellan gum system described here is the first one that exhibits a three-stage gelation mechanism for bioprinting. First, almost instant formation of a loose, physically crosslinked gellan network arrests the flow of gel directly after plotting, which is manifested as the yield stress. After that, the plotted structure further increases in strength and stiffness as the gelatine cools and forms a thermal gel. This process, which takes place over the course of minutes, prevents any noticeable deformation caused by gravity and therefore enables the construction of large and tall structures. Finally, UV-initiated crosslinking of the gelMA component fixes the shape irreversibly so it can be handled, cultured at $37{ }^{\circ} \mathrm{C}$ while submerged in culture media, and potentially mechanically loaded in a bioreactor to stimulate encapsulated cells for improved tissue regeneration. ${ }^{26}$ By adjustment of the dispensing rate relative to the stage speed, filaments of a wide range of diameters can be plotted. Constructs can be fabricated according to computed designs either in solid gel or porous, allowing perfusion and fast exchange of solutes.

The addition of gellan gum was shown to not affect the gel microstructure or cell viability, even when PBS was replaced by isotonic mannose for up to one hour. Gels with higher gellan gum concentrations showed higher compressive moduli and less swelling behaviour.

In conclusion, to our knowledge we are the first group to show that the addition of small quantities of gellan gum to gelatine-methacrylamide hydrogels and tailoring of salt concentration enables additive manufacturing with high shape fidelity without compromising cell viability.

\section{Experimental}

\section{GelMA synthesis}

Gelatine (Sigma Aldrich, type A from porcine skin, $300 \mathrm{~g}$ Bloom) was reacted with methacrylic anhydride (Sigma Aldrich 92\%) at $0.6 \mathrm{~g}$ anhydride per $\mathrm{g}$ gelatine in PBS at $10 \%$ at $50{ }^{\circ} \mathrm{C}$ for one hour, followed by removal of excess anhydride by centrifugation and decanting and dialysis (cellulose membrane with 12-14 $\mathrm{kDa}$ cut off, Sigma Aldrich) at $40{ }^{\circ} \mathrm{C}$ for $24 \mathrm{~h}$ against demineralised water (refreshed twice), freeze-dried and stored at $-20{ }^{\circ} \mathrm{C}$ until further use. Irgacure 2959 (gift from BASF) was recrystallised from water and dissolved in water at $70{ }^{\circ} \mathrm{C}$ for $5 \mathrm{~min}$, final concentration $0.05 \%$. GelMA, low-acyl gellan gum (Gelzan Gelrite, Sigma Aldrich), D-(+)-mannose (Sigma Aldrich, 5.4\% for isotonic solution), PBS were dissolved separately in water and mixed in the desired ratio at $90{ }^{\circ} \mathrm{C}$. All concentrations are in $\%$ $\mathrm{w} / \mathrm{v}$ (or $\mathrm{g}$ per $100 \mathrm{~mL}$ water).

\section{Viscosity measurements}

Viscosity measurements were performed at $37^{\circ} \mathrm{C}$ on an AR-G2 rheometer (TA Instruments) fitted with a cone-and-plate attachment. The yield stress was measured by unconfined compression on non-cured cylindrical $(6 \times 2 \mathrm{~mm} D \times h)$ injection-moulded gels at $37{ }^{\circ} \mathrm{C}$ in air at $0.01 \mathrm{~mm} \mathrm{~s}^{-1}$ on an Instron Microtester 5848 (5 N load cell).

\section{Gel plotting}

Gel plotting was performed at $34{ }^{\circ} \mathrm{C}$ on a custom-made BioExtruder bioprinter using a stepper motor driven xyz-stage and a syringe pump designed to fit disposable $5 \mathrm{~mL}$ syringes. Cell-laden gel constructs were prepared on a SysEng BioScaffolder bioprinter inside a laminar air flow cabinet, otherwise using the same materials and conditions. Gels for physical 
characterisation and the cell experiment were casted in a $50 \times 4$ $\times 2 \mathrm{~mm}$ Teflon mould, covered with a glass microscope slide, exposed to $365 \mathrm{~nm} \mathrm{UV} \mathrm{(2.6} \mathrm{mW} \mathrm{cm}^{-2}$ ) for $15 \mathrm{~min}$ (UVP CL-1000) and cut in 6 equal pieces using a scalpel blade. Plotted gel structures were UV cured for 30 min while immersed in a $0.1 \%$ Irgacure 2959 solution in PBS.

\section{Cell viability experiment}

Suspensions of MC3T3 pre-osteoblasts in gel precursor solutions were UV cured for $15 \mathrm{~min}$ and incubated overnight in Dulbecco's Modified Eagle's Medium (DMEM) supplemented with $10 \%$ foetal bovine serum and $1 \%$ penicillin/streptomycin, then in DMEM + 8\% Alamar Blue (Invitrogen) for $3 \mathrm{~h}$ after which the fluorescence was measured in a black 96 well plate (Corning) using a POLARStar OPTIMA plate reader (BMG Labtech) at excitation $544 \mathrm{~nm} /$ emission $590 \mathrm{~nm}$. For plotting, chondrocytes of the ATDC5 cell line were resuspended in isotonic mannose at $37^{\circ} \mathrm{C}$, then mixed with gelMA/gellan of $50{ }^{\circ} \mathrm{C}$ using a gel pippet (Gilson) and transferred to a $3 \mathrm{~mL}$ syringe for plotting. Constructs plotted at $34{ }^{\circ} \mathrm{C}$ were UV-cured for $15 \mathrm{~min}$ and cultured in DMEM for 4 days prior to 20 min staining with $4 \mathrm{mM}$ calcein AM (live) and $2 \mathrm{mM}$ EtBr (dead) in PBS at $37{ }^{\circ} \mathrm{C}$, and visualised on an Olympus BX51 microscope.

\section{Characterisation of physical properties}

Cell-free gels were compression tested while immersed in PBS at $37^{\circ} \mathrm{C}$ at $0.01 \mathrm{~mm} \mathrm{~s}^{-1}$ on an Instron Microtester 5848 (5 N load cell). After $24 \mathrm{~h}$ in PBS at $37{ }^{\circ} \mathrm{C}$ gels were blotted dry and weighed; effective swelling was defined as swollen weight divided by initial weight.

\section{Imaging}

A Scanco $\mu \mathrm{CT}$ scanner was employed at $55 \mathrm{kV} / 145 \mu \mathrm{A}$ for structural characterisation of porous plotted gel structures. For scanning electron microscopy imaging, samples were fixed in $3 \%$ glutaraldehyde in $0.1 \mathrm{M}$ sodium cacodylate buffer ( $\mathrm{pH} 7.4$ ) for $24 \mathrm{~h}$ at $4{ }^{\circ} \mathrm{C}$, then post-fixed in $1 \%$ osmium tetroxide for 45 min. Samples were then dehydrated in an ascending ethanol series $(50,70,90,100 \%$ and $100 \%$ again) before being critically point dried in $\mathrm{CO}_{2}$. A $10 \mathrm{~nm}$ sputter coating of gold was deposited (Leica EM SCD005 sputter coater) prior to examination using a FEI Quanta 3D Focused Ion Beam SEM, operating in $\mathrm{HV}$ mode at $10 \mathrm{kV}$. Cell-laden gels were also stained with 10 $\mu \mathrm{g} \mathrm{mL}{ }^{-1}$ fluorescein diacetate (stains live cells) and $5 \mu \mathrm{g} \mathrm{mL}$ propidium iodide (stains dead cells) for $5 \mathrm{~min}$ and imaged on a Leica SP5 confocal microscope.

\section{Conclusions}

A novel bioink based on gelatine-methacrylamide and gellan gum has been developed for robotic dispensing techniques. Rheological properties were tuned through tailored salt concentrations, whilst maintaining high cell viability. This bioink provides a promising platform for additive tissue manufacturing.

\section{Acknowledgements}

The research leading to these results has received funding from the European Community's Seventh Framework Programme (FP7/2007-2013) under grant agreements no. 309962 (HydroZONES) and no. 272286 (PrintCART Marie Curie Fellowship PIOF-GA-2010) and the Australian Research Council, Future Fellowship Grant (FT110101117) as well as the Institute For Advanced Studies (IAS), Technical University Munich. We thank Prof. Paulo Bartólo (IP Leiria, Portugal) and Dr Tim Woodfield for collaborative work on development of equipment for hydrogel bioprinting.

\section{Notes and references}

1 ASTM-International, in ASTM Standard F2792-10, West Conshohocken, PA, 2010.

2 I. Gibson, D. W. Rosen and B. Stucker, Additive Manufacturing Technologies: Rapid Prototyping to Direct Digital Manufacturing, Springer-Verlag Berlin, Berlin, 2010.

3 S. Singare, Q. Lian, W. P. Wang, J. Wang, Y. X. Liu, D. C. Li and B. H. Lu, Rapid Prototyping Journal, 2009, 15, 19-23.

4 L. Vrielinck, C. Politis, S. Schepers, M. Pauwels and U. Naert, Int. J. Oral Maxillofac. Surg., 2003, 32, 7-14.

5 F. P. W. Melchels, P. S. Wiggenhauser, D. Warne, M. Barry, F. R. Ong, W. S. Chong, D. W. Hutmacher and J. T. Schantz, Biofabrication, 2011, 3, 034114.

6 F. P. W. Melchels, M. A. N. Domingos, T. J. Klein, J. Malda, P. J. Bartolo and D. W. Hutmacher, Prog. Polym. Sci., 2012, 37, 1079-1104.

7 C. Ferris, K. Gilmore, G. Wallace and M. Panhuis, Appl. Microbiol. Biotechnol., 2013, 97, 4243-4258.

8 C. M. Smith, A. L. Stone, R. L. Parkhill, R. L. Stewart, M. W. Simpkins, A. M. Kachurin, W. L. Warren and S. K. Williams, Tissue Eng., 2004, 10, 1566-1576.

9 A. Skardal, J. Zhang, L. McCoard, X. Xu, S. Oottamasathien and G. D. Prestwich, Tissue Eng., Part A, 2010, 16, 2675-2685.

10 Y. Yan, X. Wang, Y. Pan, H. Liu, J. Cheng, Z. Xiong, F. Lin, R. Wu, R. Zhang and Q. Lu, Biomaterials, 2005, 26, 5864-5871.

11 D. L. Cohen, E. Malone, H. Lipson and L. J. Bonassar, Tissue Eng., 2006, 12, 1325-1335.

12 N. E. Fedorovich, I. Swennen, J. Girones, L. Moroni, C. A. van Blitterswijk, E. Schacht, J. Alblas and W. J. A. Dhert, Biomacromolecules, 2009, 10, 1689-1696.

13 R. Censi, W. Schuurman, J. Malda, G. di Dato, P. E. Burgisser, W. J. A. Dhert, C. F. van Nostrum, P. di Martino, T. Vermonden and W. E. Hennink, Adv. Funct. Mater., 2011, 21, 1833-1842.

14 S. Khalil and W. Sun, J. Biomech. Eng., 2009, 131.

15 D. Seliktar, Science, 2012, 336, 1124-1128.

16 S. Ahn, Y. Kim, H. Lee and G. Kim, J. Mater. Chem., 2012, 22, 15901.

17 H.-J. Lee, S.-H. Ahn and G. H. Kim, Chem. Mater., 2012, 24, 881.

18 H. Lee, S. Ahn, L. J. Bonassar and G. Kim, Macromol. Rapid Commun., 2012, 34, 142.

19 J. M. Dang and K. W. Leong, Adv. Drug Delivery Rev., 2006, 58, 487. 
20 A. I. Van den Bulcke, B. Bogdanov, N. De Rooze, E. H. Schacht, M. Cornelissen and H. Berghmans, Biomacromolecules, 2000, 1, 31-38.

21 H. Aubin, J. W. Nichol, C. B. Hutson, H. Bae, A. L. Sieminski, D. M. Cropek, P. Akhyari and A. Khademhosseini, Biomaterials, 2010, 31, 6941-6951.

22 Y. C. Chen, R. Z. Lin, H. Qi, Y. Z. Yang, H. J. Bae, J. M. MeleroMartin and A. Khademhosseini, Adv. Funct. Mater., 2012, 22, 2027-2039.

23 R. Gauvin, Y. C. Chen, J. W. Lee, P. Soman, P. Zorlutuna, J. W. Nichol, H. Bae, S. C. Chen and A. Khademhosseini, Biomaterials, 2012, 33, 3824-3834.

24 J. W. Nichol, S. T. Koshy, H. Bae, C. M. Hwang, S. Yamanlar and A. Khademhosseini, Biomaterials, 2010, 31, 5536-5544.

25 W. Schuurman, P. A. Levett, M. W. Pot, P. R. van Weeren, W. J. A. Dhert, D. W. Hutmacher, F. P. W. Melchels, T. J. Klein and J. Malda, Macromol. Biosci., 2013, 13, 551-561.

26 T. Billiet, E. Gevaert, T. De Schryver, M. Cornelissen and P. Dubruel, Biomaterials, 2013, 35, 49.

27 J. T. Oliveira, L. Martins, R. Picciochi, I. B. Malafaya, R. A. Sousa, N. M. Neves, J. F. Mano and R. L. Reis, J. Biomed. Mater. Res., Part A, 2010, 93, 852-863.
28 W. Herschel and R. Bulkley, Kolloid-Z., 1926, 39, 291-300.

29 R. J. Shipley, G. W. Jones, R. J. Dyson, B. G. Sengers, C. L. Bailey, C. J. Catt, C. P. Please and J. Malda, J. Theor. Biol., 2009, 259, 489-502.

30 P. Carmeliet, Nat. Med., 2000, 6, 389-395.

31 J. C. Reichert, A. Cipitria, D. R. Epari, S. Saifzadeh, P. Krishnakanth, A. Berner, M. A. Woodruff, H. Schell, M. Mehta, M. A. Schuetz, G. N. Duda and D. W. Hutmacher, Sci. Transl. Med., 2012, 4, 141ra93.

32 A. J. Vander, J. H. Sherman and D. S. Luciano, Human Physiology, McGraw-Hill, New York, 1985.

33 B. Parekkadan, P. Sethu, D. Van Poll, M. L. Yarmush and M. Toner, Tissue Eng., 2007, 13, 2465-2474.

34 T. Y. Lee, C. A. Guymon, E. S. Jonsson and C. E. Hoyle, Polymer, 2004, 45, 6155-6162.

35 R. A. Rezende, P. J. Bartolo, A. Mendes and R. Maciel, J. Appl. Polym. Sci., 2009, 113, 3866-3871.

36 Y. H. Gong, C. M. Wang, R. C. Lai, K. Su, F. Zhang and D. A. Wang, J. Mater. Chem., 2009, 19, 1968-1977.

37 J. Malda, J. Visser, F. P. W. Melchels, T. Jungst, W. E. Hennink, W. J. Dhert, J. Groll and D. W. Hutmacher, Adv. Mater., 2013, 25, 5011-5028. 